ANNALES

POLONICI MATHEMATICI

$83.3(2004)$

\title{
On the existence of solutions of nonlinear integral equations in Banach spaces and Henstock-Kurzweil integrals
}

\author{
by Aneta Sikorska-Nowak (Poznań)
}

\begin{abstract}
We prove some existence theorems for nonlinear integral equations of the Urysohn type $x(t)=\varphi(t)+\lambda \int_{0}^{a} f(t, s, x(s)) d s$ and Volterra type $x(t)=\varphi(t)+$ $\int_{0}^{t} f(t, s, x(s)) d s, t \in I_{a}=[0, a]$, where $f$ and $\varphi$ are functions with values in Banach spaces. Our fundamental tools are: measures of noncompactness and properties of the Henstock-Kurzweil integral.
\end{abstract}

1. Introduction. The Henstock-Kurzweil integral encompasses the Newton, Riemann and Lebesgue integrals ([8], [9], [14]). A particular feature of this integral is that integrals of highly oscillating functions such as $F^{\prime}(t)$, where $F(t)=t^{2} \sin t^{-2}$ on $(0,1]$ and $F(0)=0$, can be defined. This integral was introduced by Henstock and Kurzweil independently in 1957-58 and has since proved useful in the study of ordinary differential equations ([6], [7], [13], [17], [18]).

It is well known that Henstock's Lemma plays an important role in the theory of the Henstock-Kurzweil integral in the real-valued case. On the other hand, in connection with the Henstock-Kurzweil integral for Banach space valued functions, S. S. Cao pointed out in [5] that Henstock's Lemma holds for the case of finite dimensions, but it does not always hold in infinite dimensions.

In this paper we will use the HL integral which satisfies Henstock's Lemma and which is more general than the Bochner integral.

Let $E$ be a Banach space, $E_{1}$ a separable Banach space, and let $I_{a}=[0, a]$, $a \in \mathbb{R}_{+}$. We will prove some existence theorems for the Urysohn integral

2000 Mathematics Subject Classification: Primary 34G20, 28B05, 45D05.

Key words and phrases: existence of solution, measure of noncompactness, nonlinear Volterra integral equation, nonlinear Urysohn integral equation, Henstock-Kurzweil integral, HL integral. 
equation

$$
x(t)=\varphi(t)+\lambda \int_{0}^{a} f(t, s, x(s)) d s, \quad \lambda>0, t \in I_{a},
$$

and for the Volterra integral equation

$$
x(t)=\varphi(t)+\int_{0}^{t} f(t, s, x(s)) d s, \quad t \in I_{a},
$$

where $f$ and $\varphi$ are functions with values in the Banach space $E$ (or in the separable Banach space $E_{1}$ ) and the integral is the HL integral. Our fundamental tools are the Kuratowski and Hausdorff measures of noncompactness ([12], see also [3]), and the properties of the HL integral ([5], [17]).

The result presented in this paper contains several results concerning the Bochner integral (e.g. Januszewski [10], Krzyśka [11]) and the HenstockKurzweil integral for real-valued functions (Bugajewski [4]).

For any bounded subset $A$ of $E$ we denote by $\alpha(A)$ the Kuratowski measure of noncompactness of $A$, i.e. the infimum of all $\varepsilon>0$ such that there exists a finite covering of $A$ by sets of diameter smaller than $\varepsilon$.

For any bounded subset $A$ of $E$ we denote by $\alpha_{1}(A)$ the Hausdorff measure of noncompactness of $A$, i.e. the infimum of all $\varepsilon>0$ such that $A$ can be covered by a finite number of balls of radius smaller than $\varepsilon$.

The properties of the measures of noncompactness $\gamma=\alpha, \alpha_{1}$ are:

(i) if $A \subset B$ then $\gamma(A) \leq \gamma(B)$;

(ii) $\gamma(A)=\gamma(\bar{A})$, where $\bar{A}$ denotes the closure of $A$;

(iii) $\gamma(A)=0$ if and only if $A$ is relatively compact;

(iv) $\gamma(A \cup B)=\max \{\gamma(A), \gamma(B)\}$;

(v) $\gamma(\lambda A)=|\lambda| \gamma(A)(\lambda \in \mathbb{R})$;

(vi) $\gamma(A+B) \leq \gamma(A)+\gamma(B)$;

(vii) $\gamma(\operatorname{conv} A)=\gamma(A)$;

(viii) $\alpha_{1}(A) \leq \alpha(A) \leq 2 \alpha_{1}(A)$.

We will need the following lemma:

LEMMA $1.1([2])$. Let $H \subset C\left(I_{a}, E\right)$ be a family of strongly equicontinuous functions. Then

$$
\alpha_{C}(H)=\sup _{t \in I_{a}} \alpha(H(t))=\alpha\left(H\left(I_{a}\right)\right),
$$

where $\alpha_{C}(H)$ denotes the measure of noncompactness in $C\left(I_{a}, E\right)$ and the function $t \mapsto \alpha(H(t))$ is continuous.

2. Henstock-Kurzweil integral in Banach spaces. In this part we define the Henstock-Kurzweil integral in a Banach space and we give some properties of this integral. 
Definition $2.1([8])$. Let $\delta(\cdot)$ be a positive function defined on the interval $[a, b]$. A tagged interval $(x,[c, d])$ consists of an interval $[c, d] \subset[a, b]$ and a point $x \in[c, d]$. The tagged interval $(x,[c, d])$ is subordinate to $\delta$ if $[c, d] \subset[x-\delta(x), x+\delta(x)]$. The letter $P$ will be used to denote finite collections of nonoverlapping tagged intervals. Let

$$
P=\left\{\left(s_{i},\left[c_{i}, d_{i}\right]\right): 1 \leq i \leq n\right\}, \quad n \in \mathbb{N}
$$

be such a collection in $[a, b]$.

(i) The points $\left\{s_{i}: 1 \leq i \leq n\right\}$ are called the tags of $P$.

(ii) The intervals $\left\{\left[c_{i}, d_{i}\right]: 1 \leq i \leq n\right\}$ are called the intervals of $P$.

(iii) If $\left\{\left(s_{i},\left[c_{i}, d_{i}\right]\right): 1 \leq i \leq n\right\}$ is subordinate to $\delta$ for each $i$, then we say that $P$ is sub $\delta$.

(iv) If $[a, b]=\bigcup_{i=1}^{n}\left[c_{i}, d_{i}\right]$ then $P$ is called a tagged partition of $[a, b]$.

(v) If $P$ is a tagged partition of $[a, b]$ and $P$ is sub $\delta$, then $P$ is sub $\delta$ on $[a, b]$.

(vi) If $f:[a, b] \rightarrow E$ then $f(P):=\sum_{i=1}^{n} f\left(s_{i}\right)\left(d_{i}-c_{i}\right)$.

(vii) If $F$ is a function defined on the subintervals of $[a, b]$, then

$$
F(P)=\sum_{i=1}^{n} F\left(\left[c_{i}, d_{i}\right]\right)=\sum_{i=1}^{n}\left(F\left(d_{i}\right)-F\left(c_{i}\right)\right) .
$$

Definition $2.2([5])$. The function $f:[a, b] \rightarrow E$ is Henstock-Kurzweil integrable on $[a, b](f \in \operatorname{HK}([a, b], E))$ if there exists a vector $z$ in $E$ with the following property: for each $\varepsilon>0$ there exists a positive function $\delta$ on $[a, b]$ such that $\|f(P)-z\|<\varepsilon$ whenever $P$ is $\operatorname{sub} \delta$ on $[a, b]$. The function $f$ is Henstock-Kurzweil integrable on $A \subset[a, b]$ if $f \chi_{A}$ is Henstock-Kurzweil integrable on $[a, b]$. The vector $z$ is the Henstock-Kurzweil integral of $f$.

We remark that this definition includes the generalized Riemann integral defined by Gordon in [8].

Definition $2.3([5])$. Let $f$ be Henstock-Kurzweil integrable on $[a, b]$. Then the function $F(t)=\int_{a}^{t} f$, which is defined on subintervals of $[a, b]$, is called the primitive of $f$.

Definition $2.4([5])$. A function $f:[a, b] \rightarrow E$ is $H L$ integrable on $[a, b]$ $(f \in \operatorname{HL}([a, b], E))$ if there exists a function $F:[a, b] \rightarrow E$ with the following property: given $\varepsilon>0$ there exists a positive function $\delta(\cdot)$ on $[a, b]$ such that if $P=\left\{\left(s_{i},\left[c_{i}, d_{i}\right]: 1 \leq i \leq n\right\}\right.$ is a tagged partition of $[a, b]$ sub $\delta$, then

$$
\sum_{i=1}^{n}\left\|f\left(s_{i}\right)\left(d_{i}-c_{i}\right)-F\left(\left[c_{i}, d_{i}\right]\right)\right\|<\varepsilon .
$$

We note that by the triangle inequality $f \in \operatorname{HL}([a, b], E)$ implies $f \in$ $\operatorname{HK}([a, b], E)$. In general, the converse is not true. For real-valued functions, the two integrals are equivalent. 
We now recall Henstock's Lemma for real-valued Henstock-Kurzweil integrable functions (for the proof, see [14, Theorem 3.7]).

TheORem 2.5 (Henstock's Lemma). If $f$ is Henstock-Kurzweil integrable on $[a, b]$ with primitive $F$, then for every $\varepsilon>0$ there exists $\delta(\cdot)>0$ such that for any tagged partition $P=\left\{\left(s_{i},\left[c_{i}, d_{i}\right]\right): 1 \leq i \leq n\right\}$ of $[a, b]$ sub $\delta$, we have

$$
\sum_{i=1}^{n}\left|f\left(s_{i}\right)\left(d_{i}-c_{i}\right)-F\left(\left[c_{i}, d_{i}\right]\right)\right|<\varepsilon .
$$

Theorem 2.5 says that in the definition of the Henstock-Kurzweil integral for real-valued functions ([8]), we may put the absolute value sign $|\cdot|$ inside the summation sign $\sum$. We know ([5]) that this is no longer true if we replace | | | with $\|\cdot\|$, i.e. Henstock's Lemma is not satisfied by Henstock-Kurzweil integrable Banach-valued functions. By the definition of HL integral, an HL integrable function with primitive $F$ satisfies Henstock's Lemma with $|\cdot|$ replaced $\|\cdot\|$.

Definition $2.6([8])$. A family $\mathcal{F}$ of functions $F$ is said to be uniformly absolutely continuous in the restricted sense on $A$, for short uniformly $A C_{*}(A)$, if for every $\varepsilon>0$ there exists $\eta>0$ such that for every $F$ in $\mathcal{F}$ and for every finite or infinite sequence of nonoverlapping intervals $\left\{\left[a_{i}, b_{i}\right]\right\}$ with $a_{i}, b_{i} \in A$ and satisfying $\sum_{i}\left|b_{i}-a_{i}\right|<\eta$, we have $\sum_{i} \omega\left(F,\left[a_{i}, b_{i}\right]\right)<\varepsilon$, where $\omega\left(F,\left[a_{i}, b_{i}\right]\right)$ denotes the oscillation of $F$ over $\left[a_{i}, b_{i}\right]$.

A family $\mathcal{F}$ of functions $F$ is said to be uniformly generalized absolutely continuous in the restricted sense on $[a, b]$, or uniformly $A C G_{*}$, if $[a, b]$ is the union of a sequence of closed sets $A_{i}$ such that on each $A_{i}$ the function $F$ is uniformly $A C_{*}\left(A_{i}\right)$.

For the Henstock-Kurzweil integral, in particular for the HL integral, we have the following theorems.

Theorem $2.7([5])$. Let $f:[a, b] \rightarrow E$. If $f=0$ almost everywhere on $[a, b]$, then $f$ is $H L$ integrable on $[a, b]$ and $\int_{a}^{b} f(t) d t=0$.

Theorem $2.8([5])$. Let $f:[a, b] \rightarrow E$ be $H L$ integrable on $[a, b]$ and let $F(x)=\int_{a}^{x} f(t) d t$ for each $x \in[a, b]$. Then

(i) $F$ is continuous on $[a, b]$,

(ii) $F$ is differentiable almost everywhere on $[a, b]$ and $F^{\prime}=f$,

(iii) $f$ is measurable.

Theorem 2.9 ([20, Theorem 5]). Suppose that $f_{n}:[a, b] \rightarrow E, n=$ $1,2, \ldots$, is a sequence of $H L$ integrable functions satisfying the following conditions: 
(i) $f_{n}(x) \rightarrow f(x)$ almost everywhere in $[a, b]$ as $n \rightarrow \infty$;

(ii) the set of primitives of $f_{n},\left\{F_{n}(x)\right\}$, where $F_{n}(x)=\int_{a}^{x} f_{n}(s) d s$, is uniformly $A C G_{*}$ in $n$;

(iii) the primitives $F_{n}$ are equicontinuous on $[a, b]$.

Then $f$ is $H L$ integrable on $[a, b]$ and $\int_{a}^{x} f_{n} \rightarrow \int_{a}^{x} f$ uniformly on $[a, b]$ as $n \rightarrow \infty$.

We remark that this theorem for Denjoy-Bochner integrals is mentioned in [20] without proof. It is also true for HL integrals. The proof is similar to that of Theorem 7.6 in [14] (see also [19, Theorem 1.8]).

Lemma 2.10 ([17]). Let $E_{1}$ be a separable Banach space. Suppose that $V$ is a countable set of $H L$ integrable functions. Let $F=\left\{\int_{0}^{t} x(s) d s: x \in V\right.$, $\left.t \in I_{a}\right\}$ be equicontinuous, equibounded and uniformly $A C G_{*}$ on $I_{a}$. Then $\alpha_{1}\left(\int_{0}^{t} V(s) d s\right) \leq \int_{0}^{t} \alpha_{1}(V(s)) d s, t \in I_{a}$, whenever $\alpha_{1}(V(s)) \leq \varphi(s)$ for a.e. $s \in I_{a}, \varphi$ is a Lebesgue integrable function and $\alpha_{1}$ denotes the Hausdorff measure of noncompactness.

Theorem 2.11. If the function $f: I_{a} \rightarrow E$ is $H L$ integrable, then

$$
\int_{I} f(t) d t \in|I| \overline{\operatorname{conv}} f(I),
$$

where $I$ is an arbitrary subinterval of $I_{a}$ and $|I|$ is the length of $I$.

The proof is similar to that of Lemma 2.1.3 in [15]. See also [1, Theorem 10.4, p. 268].

\section{Main result}

I. The Urysohn integral equation. Now we prove an existence theorem for the problem (1.1). We will apply the following fixed point theorem.

TheORem 3.1 ([16]). Let $D$ be a closed convex subset of $E$, and let $F$ be a continuous map from $D$ into itself. If for some $x \in D$ the implication

$$
\bar{V}=\overline{\operatorname{conv}}(\{x\} \cup F(V)) \Rightarrow V \text { is relatively compact }
$$

holds for every countable subset $V$ of $D$, then $F$ has a fixed point.

We recall that a function $f: I_{a} \times I_{a} \times E \rightarrow E$ is a Carathéodory function if for each $x \in E, f(t, s, x)$ is measurable in $(t, s) \in I_{a} \times I_{a}$ and for almost all $(t, s) \in I_{a} \times I_{a}, f(t, s, x)$ is continuous with respect to $x$.

A nonnegative real-valued function $(t, s, z) \mapsto h(t, s, z)$ defined on $I_{a} \times$ $I_{a} \times E$ is a Kamke function if $h$ satisfies the Carathéodory conditions, for each fixed $t, s$ the function $z \mapsto h(t, z, s)$ is nondecreasing, and for each $q$, $0<q \leq a$, the identically zero function is the unique continuous solution of the integral equation $z(t)=\int_{0}^{t} h(t, s, z(s)) d s$ defined on $[0, q)$. 
Let $B=\left\{x \in C\left(I_{a}, E\right):\|x\| \leq\|\varphi(\cdot)\|+\lambda b\right\}$, where $b, \lambda$ are some positive numbers.

We define the operator $F_{x}$ by

$$
F_{x}(t)=\varphi(t)+\lambda \int_{0}^{a} f(t, s, x(s)) d s \quad \text { for } t \in I_{a} \text { and } x \in B,
$$

where the integral is the HL integral.

Moreover, let $F=\left\{F_{x}: x \in B\right\}$.

Theorem 3.2. Assume that for each continuous function $x: I_{a} \rightarrow E_{1}$, $f(\cdot, s, x(s))$ is $H L$ integrable, $f$ is a Carathéodory function and

$$
\alpha(f(t, s, X)) \leq h(t, s, \alpha(X)) \quad \text { for each } X \subset B, 0 \leq s \leq t \leq a,
$$

where $h$ is a Kamke function. Suppose that $F$ is equicontinuous, equibounded and uniformly $A C G_{*}$ on $I_{a}$. Then there exists a solution of the problem (1.1) on $I_{c}$ for some $0<c \leq a$ with continuous initial function $\varphi$.

Proof. By equicontinuity and equiboundedness of $F$ there exists some number $c(0<c \leq a)$ such that $\left\|\int_{0}^{c} f(t, s, x(s)) d s\right\| \leq b$ for fixed $b>0$, $t \in I_{c}$ and $x \in B$.

By our assumptions, the operator $F_{x}$ is well defined and maps $B$ into $B$ and by Theorem 2.8, $F_{x}$ is continuous. Observe that a fixed point of $F_{x}$ is a solution of the problem (1.1). Now we prove that $F_{x}$ has a fixed point using Theorem 3.1.

Suppose that $V \subset B$ is a countable set and $\bar{V}=\overline{\operatorname{conv}}\left(\{x\} \cup F_{x}(V)\right)$ is equicontinuous. Then $t \mapsto v(t)=\alpha(V(t))$ is continuous on $I_{c}$ (by Lemma 1.1).

Let us fix $t \in I_{c}$. Let $\int_{0}^{c} Z(s) d s=\left\{\int_{0}^{c} x(s) d s: x \in Z\right\}$ for any $Z \subset$ $C\left(I_{c}, E\right)$ and let $\widetilde{f}_{t}$ denote the mapping defined by $\widetilde{f}_{t}(x(s))=f(t, s, x(s))$ for each $x \in B$ and $s \in I_{c}$. Obviously, $\widetilde{f}_{t}(V(s))=f(t, s, V(s))$ and $F_{x}(V(t))=$ $\varphi(t)+\lambda \int_{0}^{c} \widetilde{f}_{t}(V(s)) d s$.

Using (3.1), Lemma 2.10 and the properties of the measure of noncompactness $\alpha$ we have

$$
\begin{aligned}
\alpha\left(F_{x}(V(t))\right. & =\alpha\left(\varphi(t)+\lambda \int_{0}^{c} \widetilde{f}_{t}(V(s)) d s\right) \leq 2 \alpha_{1}\left(\lambda \int_{0}^{c} \widetilde{f}_{t}(V(s)) d s\right) \\
& \leq 2 \lambda \int_{0}^{c} \alpha_{1}(f(t, s, V(s))) d s \leq 2 \lambda \int_{0}^{c} \alpha(f(t, s, V(s)) d s \\
& \leq 2 \lambda \int_{0}^{c} h(t, s, \alpha(V(s))) d s .
\end{aligned}
$$

Because $V=\overline{\operatorname{conv}}\left(\{x\} \cup F_{x}(V)\right)$ we have 


$$
v(t) \leq 2 \lambda \int_{0}^{c} h(t, s, v(s)) d s .
$$

Hence applying now a theorem on differential inequalities we get $v(t)=$ $\alpha(V(t))=0$. By Arzelà-Ascoli's theorem, $V$ is relatively compact. So, by Theorem 3.1, $F_{x}$ has a fixed point which is a solution of the problem (1.1).

Now we present an existence theorem for the problem (1.1) in a real Banach space $E$. Let $r(K)$ be the spectral radius of the integral operator $K$ defined by $K u(t)=\int_{0}^{a} k(t, s) u(s) d s, u \in B, t \in I_{a}$.

THEOREM 3.3. Assume that for each continuous function $x: I_{a} \rightarrow E$, $f(\cdot, s, x(s))$ is $H L$ integrable, $f$ is a Carathéodory function and there exists a function $k: I_{a} \times I_{a} \rightarrow \mathbb{R}_{+}$such that $k(t, \cdot)$ is continuous and

$$
\alpha(f(t, J, X)) \leq \sup _{s \in J} k(t, s) \alpha(X) \quad \text { for each } X \subset E \text { and } J \subset I_{a} .
$$

Suppose that $F$ is equicontinuous, equibounded and uniformly $A C G_{*}$ on $I_{a}$. Moreover, let $\lambda r(K)<1$. Then there exists a solution of the problem (1.1) on $I_{c}$ for some $0<c \leq a$ with continuous initial function $\varphi$.

Proof. By equicontinuity and equiboundedness of $F$ there exists some number $c(0<c \leq a)$ such that $\left\|\int_{0}^{c} f(t, s, x(s)) d s\right\| \leq b$ for fixed $b>0$, $t \in I_{c}$ and $x \in B$.

By our assumptions, the operator $F_{x}$ is well defined and maps $B$ into $B$, and by Theorem 2.8, $F_{x}$ is continuous. Observe that a fixed point of $F_{x}$ is a solution of the problem (1.1). Now we prove that $F_{x}$ has a fixed point using Theorem 3.1. Suppose that $V \subset B$ is a countable set and $\bar{V}=\overline{\operatorname{conv}}(\{x\} \cup$ $\left.F_{x}(V)\right)$ is equicontinuous. Then $t \mapsto v(t)=\alpha(V(t))$ is continuous on $I_{c}$ (by Lemma 1.1).

We divide the interval $I_{c}: 0=t_{0}<t_{1}<\cdots<t_{m}=c$, where $t_{i}=$ $i c / m, i=0,1, \ldots, m$. Let $V\left(\left[t_{i}, t_{i+1}\right]\right)=\left\{u(s): u \in V, t_{i} \leq s \leq t_{i+1}\right.$, $i=0,1, \ldots, m-1\}$. By Lemma 1.1 and the continuity of $v$ there exists $s_{i} \in T_{i}=\left[t_{i}, t_{i+1}\right]$ such that

$$
\alpha\left(V\left(\left[t_{i}, t_{i+1}\right]\right)\right)=\sup \left\{\alpha(V(s)): t_{i} \leq s \leq t_{i+1}\right\}=: v\left(s_{i}\right) .
$$

On the other hand, by the definition of the operator $F_{x}$ and Theorem 2.11 we have

$$
\begin{aligned}
F_{u}(t) & =\varphi(t)+\lambda \sum_{i=0}^{m-1} \int_{t_{i}}^{t_{i+1}} f(t, s, u(s)) d s \\
& \in \varphi(t)+\lambda \sum_{i=0}^{m-1}\left(t_{i+1}-t_{i}\right) \overline{\operatorname{conv}} f\left(t, T_{i}, V\left(\left[t_{i}, t_{i+1}\right]\right)\right)
\end{aligned}
$$


for each $u \in V$. Therefore

$$
F_{u}(V(t)) \subset \varphi(t)+\lambda \sum_{i=0}^{m-1}\left(t_{i+1}-t_{i}\right) \overline{\operatorname{conv}} f\left(t, T_{i}, V\left(\left[t_{i}, t_{i+1}\right]\right)\right) .
$$

Using (3.2), (3.3), and the properties of the measure of noncompactness $\alpha$ we obtain

$$
\begin{aligned}
\alpha\left(F_{u}(V(t))\right) & \leq \lambda \sum_{i=0}^{m-1}\left(t_{i+1}-t_{i}\right) \sup _{s \in T_{i}} k(t, s) \alpha\left(V\left[t_{i}, t_{i+1}\right]\right) \\
& =\lambda \sum_{i=0}^{m-1}\left(t_{i+1}-t_{i}\right) k\left(t, p_{i}\right) v\left(s_{i}\right)
\end{aligned}
$$

where $s_{i}, p_{i} \in T_{i}$, hence

$$
\begin{aligned}
\alpha\left(F_{u}(V(t))\right) \leq & \lambda \sum_{i=0}^{m-1}\left(t_{i+1}-t_{i}\right) k\left(t, p_{i}\right) v\left(p_{i}\right) \\
& +\lambda \sum_{i=0}^{m-1}\left(t_{i+1}-t_{i}\right)\left[k\left(t, p_{i}\right)\left(v\left(s_{i}\right)-v\left(p_{i}\right)\right)\right] \\
= & \lambda \sum_{i=0}^{m-1}\left(t_{i+1}-t_{i}\right) k\left(t, p_{i}\right) v\left(p_{i}\right) \\
& +\lambda \frac{c}{m} \sum_{i=0}^{m-1} k\left(t, p_{i}\right)\left(v\left(s_{i}\right)-v\left(p_{i}\right)\right) .
\end{aligned}
$$

By continuity of $v$ we have $v\left(s_{i}\right)-v\left(p_{i}\right)<\varepsilon$ and $\varepsilon \rightarrow 0$ as $m \rightarrow \infty$. So

$$
\alpha\left(F_{u}(V(t))\right)<\lambda \int_{0}^{c} k(t, s) v(s) d s+\lambda c \sup _{p \in I_{c}} k(t, p) \varepsilon .
$$

Therefore

$$
\alpha\left(F_{u}(V(t))\right) \leq \lambda \int_{0}^{c} k(t, s) v(s) d s \quad \text { for } t \in I_{c} .
$$

Since $V=\overline{\operatorname{conv}}\left(\{u\} \cup F_{u}(V)\right)$, by the property of the measure of noncompactness we have $\alpha(V(t)) \leq \alpha\left(F_{u}(V(t))\right)$ and so in view of (3.4) it follows that $v(t) \leq \lambda \int_{0}^{c} k(t, s) v(s) d s$ for $t \in I_{c}$. Because this inequality holds for every $t \in I_{c}$ and $\lambda r(K)<1$, by applying a theorem on integral inequalities, we conclude that $\alpha(V(t))=0$ for $t \in I_{c}$. Hence Arzelà-Ascoli's theorem proves that the set $V$ is relatively compact. Consequently, by Theorem 3.1, $F_{x}$ has a fixed point which is a solution of the problem (1.1).

II. The Volterra integral equation. Now we consider the integral equation (1.2). Put $B_{1}=\left\{x \in C\left(I_{a}, E\right):\|x\| \leq\|\varphi(\cdot)\|+l\right\}$, where $l$ is some 
positive number. We define the operator $G_{x}$ by

$$
G_{x}(t)=\varphi(t)+\int_{0}^{t} f(t, s, x(s)) d s \quad \text { for } t \in I_{a} \text { and } x \in B_{1},
$$

where the integral is the HL integral.

Moreover, let $G=\left\{G_{x}: x \in B_{1}\right\}$.

TheOREM 3.4. Assume that for each continuous function $x: I_{a} \rightarrow E_{1}$, $f(\cdot, s, x(s))$ is $H L$ integrable, $f$ is a Carathéodory function and

$$
\alpha(f(t, s, X)) \leq h(t, s, \alpha(X)) \quad \text { for each } X \subset B, 0 \leq s \leq t \leq a,
$$

where $h$ is a Kamke function. Suppose that $G$ is equicontinuous, equibounded and uniformly $A C G_{*}$ on $I_{a}$. Then there exists a solution of the problem (1.2) on $I_{c}$ for some $0<c \leq a$ with continuous initial function $\varphi$.

Proof. By equicontinuity and equiboundedness of $G$ there exists some number $c(0<c \leq a)$ such that $\left\|\int_{0}^{t} f(t, s, x(s)) d s\right\| \leq l$ for $t \in I_{c}$ and $x \in B_{1}$.

Let $V=\overline{\operatorname{conv}}\left(\{x\} \cup G_{x}(V)\right)$ for some bounded countable set $V \subset B_{1}$.

Analogously to the proof of Theorem 3.2 we prove that $v(t) \leq$ $2 \int_{0}^{t} f(t, s, v(s)) d s$ for $t \in I_{c}$. Hence applying now a theorem on differential inequalities we get $v(t)=\alpha(V(t))=0$. By Arzelà-Ascoli's theorem, $V$ is relatively compact. So, by Theorem $3.1, G_{x}$ has a fixed point which is a solution of the problem (1.1).

Now, let $r(K)$ be the spectral radius of the integral operator $K$ defined by $K u(t)=\int_{0}^{t} k(t, s) u(s) d s, u \in B_{1}, t \in I_{a}$.

For a real Banach space $E$ we have the following theorem.

Theorem 3.5. Assume that for each continuous function $x: I_{a} \rightarrow E$, $f(\cdot, s, x(s))$ is $H L$ integrable, $f$ is a Carathéodory function and there exists a function $k: I_{a} \times I_{a} \rightarrow \mathbb{R}_{+}$such that $k(t, \cdot)$ is continuous and

$$
\alpha(f(t, J, X)) \leq \sup _{s \in J} k(t, s) \alpha(X) \quad \text { for each } X \subset E \text { and } J \subset I_{a} .
$$

Suppose that $G$ is equicontinuous, equibounded and uniformly $A C G_{*}$ on $I_{a}$. Moreover, let $r(K)<1$. Then there exists a solution of the problem (1.2) on $I_{c}$ for some $0<c \leq a$ with continuous initial function $\varphi$.

Proof. By equicontinuity and equiboundedness of $G$ there exists some number $c(0<c \leq a)$ such that $\left\|\int_{0}^{t} f(t, s, x(s)) d s\right\| \leq l$ for $t \in I_{c}$ and $x \in B_{1}$.

Let $V=\overline{\operatorname{conv}}\left(\{x\} \cup G_{x}(V)\right)$ for some bounded countable set $V \subset B_{1}$.

Analogously to the proof of Theorem 3.3 we divide the interval $[0, t]$ (for fixed $t \in I_{c}$ ) into $m$ parts $0=t_{0}<t_{1}<\cdots<t_{m}=t$, where $t_{i}=i t / m$, 
$i=0,1, \ldots, m$, and we prove that $v(t) \leq \int_{0}^{t} k(t, s) v(s) d s$ for $t \in I_{c}$. Because this inequality holds for every $t \in I_{c}$ and $r(K)<1$, by applying a theorem on integral inequalities, we conclude that $\alpha(V(t))=0$ for $t \in I_{c}$. Hence Arzelà-Ascoli's theorem proves that the set $V$ is relatively compact. Consequently, by Theorem 3.1, $G_{x}$ has a fixed point which is a solution of the problem (1.2).

REMARK. Let $S$ be the set of solutions of the problems (1.1) and (1.2) and $S_{n} \subset S$ be a sequence of solutions. As $S_{n}=F\left(S_{n}\right)$, by repeating the calculation in the proof of Theorems 3.2 (or Theorem 3.3) and 3.4 (or Theorem 3.5), where $V=S_{n}$, we can show that $S$ is compact.

\section{References}

[1] A. Alexiewicz, Functional Analysis, PWN, Warszawa, 1969 (in Polish).

[2] A. Ambrosetti, Un teorema di esistenza per le equazioni differenziali negli spazi di Banach, Rend. Sem. Univ. Padova 39 (1967), 349-361.

[3] J. Banaś and K. Goebel, Measures of Noncompactness in Banach Spaces, Lecture Notes in Pure and Appl. Math. 60, Dekker, New York and Basel, 1980.

[4] D. Bugajewski, On the Volterra integral equation and the Henstock-Kurzweil integral, Math. Rommanica 9 (1998), 141-145.

[5] S. S. Cao, The Henstock integral for Banach valued functions, SEA Bull. Math. 16 (1992), 36-40.

[6] T. S. Chew, On Kurzweil generalized ordinary differential equations, J. Differential Equations 76 (1988), 286-293.

[7] T. S. Chew and F. Flordelija, On $x^{\prime}=f(t, x)$ and Henstock-Kurzweil integrals, Differential Integral Equations 4 (1991), 861-868.

[8] R. A. Gordon, The Integrals of Lebesgue, Denjoy, Perron and Henstock, Amer. Math. Soc., Providence, RI, 1994.

[9] R. Henstock, The General Theory of Integration, Oxford Math. Monographs, Clarendon Press, Oxford, 1991.

[10] J. Januszewski, On the existence of continuous solutions of nonlinear integral equations in Banach spaces, Comment. Math. 30 (1990), 85-92.

[11] S. Krzyśka, On the existence of continuous solutions of Urysohn and Volterra integral equations in Banach spaces, Demonstratio Math. 2 (1995), 353-359.

[12] K. Kuratowski, Topologie, PWN-Polish Sci. Publ., Warszawa, 1958.

[13] J. Kurzweil, Generalized ordinary differential equations and continuous dependence on a parametr, Czechoslovak Math. J. 7 (1957), 642-659.

[14] P. Y. Lee, Lanzhou Lectures on Henstock Integration, Ser. Real Anal. 2, World Sci., Singapore, 1989.

[15] R. H. Martin, Nonlinear Operators and Differential Equations in Banach Spaces, Krieger, Melbourne, FL, 1987.

[16] H. Mönch, Boundary value problems for nonlinear ordinary differential equations of second order in Banach spaces, Nonlinear Anal. 4 (1980), 985-999.

[17] A. Sikorska-Nowak, Retarded functional differential equations in Banach spaces and Henstock-Kurzweil integrals, Demonstratio Math. 35 (2002), 49-60. 
[18] A. Sikorska and I. Kubiaczyk, Differential equations in Banach spaces and Henstock-Kurzweil integrals, Discuss. Math. Differ. Incl. 19 (1999), 35-43.

[19] A. P. Solodov, On condition of differentiability almost everywhere for absolutely continuous Banach-valued function, Moscow Univ. Math. Bull. 54 (1999), 29-32.

[20] G. Ye, P. Y. Lee and C. Wu, Convergence theorems of the Denjoy-Pettis and Denjoy-Dunford integrals, SEA Bull. Math. 23 (1999), 135-140.

Faculty of Mathematics and Computer Science

Adam Mickiewicz University

Umultowska 87, 61-614 Poznań, Poland

E-mail: anetas@amu.edu.pl

Reçu par la Rédaction le 27.10.2003

Révisé le 19.7.2004 